\title{
The Antares Neutrino Telescope and Multi-Messenger Astronomy
}

\author{
Thierry PRADIER*, on behalf of the ANTARES Collaboration \\ * University of Strasbourg (France) \& Institut Pluridisciplinaire Hubert Curien
}

\begin{abstract}
AnTARES is currently the largest neutrino telescope operating in the Northern Hemisphere, aiming at the detection of high-energy neutrinos from astrophysical sources. Such observations would provide important clues about the processes at work in those sources, and possibly help solve the puzzle of ultra-high energy cosmic rays. In this context, ANTAREs is developing several programs to improve its capabilities of revealing possible spatial and/or temporal correlations of neutrinos with other cosmic messengers: photons, cosmic rays and gravitational waves. The neutrino telescope and its most recent results are presented, together with these multi-messenger programs.
\end{abstract}

\section{Introduction}

Astroparticle physics has entered an exciting period with the recent development of experimental techniques that have opened new windows of observation of the cosmic radiation in all its components: photons, cosmic rays, but also gravitational waves and high energy neutrinos, that could be detected both by IceCube [1] and ANTARES.

The advantage of using neutrinos as new messengers lies firstly on their weak interaction cross-section ; unlike protons or $\gamma$, they provide a cosmological-range unaltered information from the very heart of their sources. Secondly, charged particles are deflected by magnetic fields. Neutrinos on the other hand point directly to their sources and exact production site.

The neutrinos ANTARES is aiming at are typically $\mathrm{TeV}$ neutrinos from AGNs or galactic sources (microquasars), 30 orders of magnitude lower in flux than solar neutrinos. The detection of those specific neutrinos requires under water/ice instruments, or alternatively acoustic/radio techniques in the PeV-EeV range and air showers arrays above $1 \mathrm{EeV}$. In spite of efforts in those various energy ranges, since the detection of the MeV neutrino burst from SN 1987A by KAMIOKANDE/BAKSAN/IMB/MONT-BlAnC 2] no astrophysical source for neutrinos above a few $\mathrm{GeV}$ has ever been identified.

Sources for $\mathrm{TeV} \nu$ are typically compact objects (neutron stars/black holes), from which often emerge relativistic plasma jets with a still unclear composition - purely

$\ddagger$ http://antares.in2p3.fr 
leptonic or with some hadronic component. Most of these sources have already been extensively studied from radio wavelengths up to $\gamma$-rays. These photons can be produced by $e^{-}$via inverse compton effect (on ambient photon field)/synchrotron radiation, or by protons/nuclei via photoproduction of $\pi^{0} / \pi^{ \pm}$:

$$
\begin{aligned}
& p / A+p / \gamma \longrightarrow \pi^{0} \pi^{ \pm} \\
& \text {where } \pi^{0} \rightarrow \gamma \gamma \text { and } \pi^{ \pm} \rightarrow \nu_{\mu} \mu, \text { with } \mu \rightarrow \nu_{\mu} \nu_{e} e
\end{aligned}
$$

In the former scenario, no neutrinos are produced, whereas in the latter, the neutrino flux is directly related to the gamma flux: a $\mathrm{TeV}$ neutrino detection from gamma sources would then yield a unique way to probe the inner processes of the most powerful events in the universe. Several hints exist which indicates that hadrons could be accelerated up to very high energies. Firstly, the combined radio, X-rays and $\gamma$ rays observations of the shell-type supernova remnant RX J1713.7-3946 [3] favour the production of photons via $\pi^{0}$ decay (figure 1, left). Secondly, the correlations between $\mathrm{X}$ and $\gamma$ for the Blazar 1ES1959+650 [4] prove the existence of $\gamma$ flares not visible in $\mathrm{X}$ (figure 1, right), which is difficult to account for in purely leptonic models.
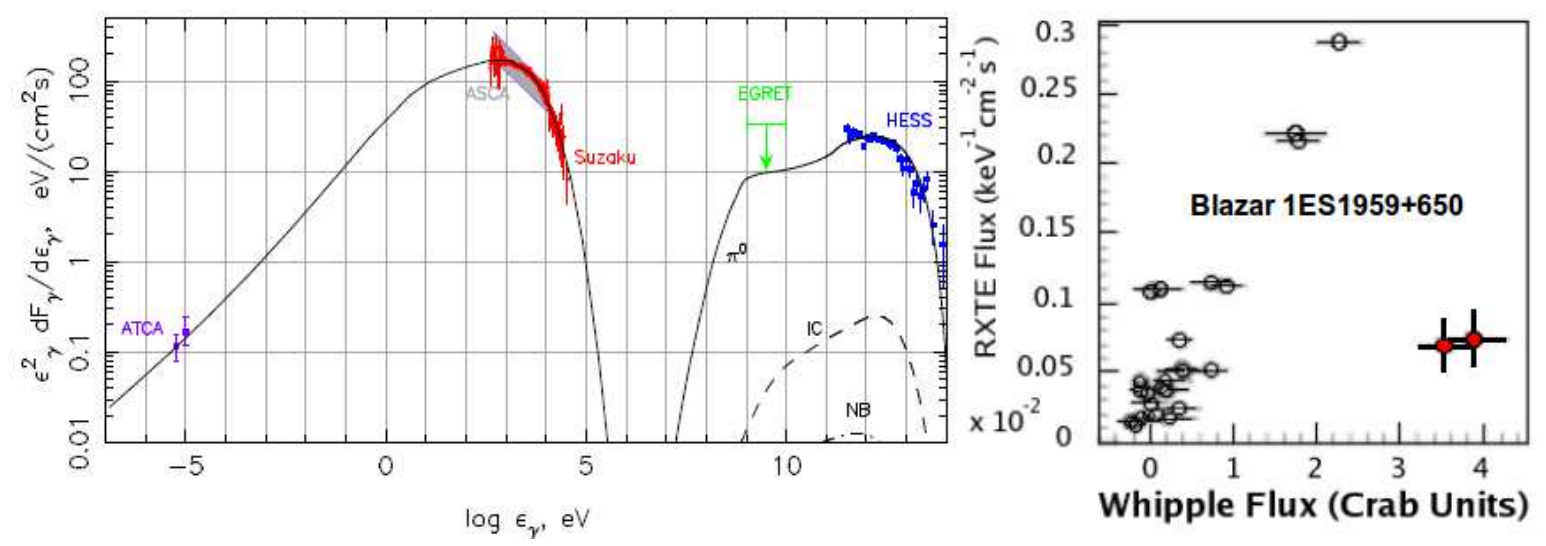

Figure 1. Left: Multiwavelength observations of the SNR RXJ 1713.7-39; the solid curve at energies above $10^{7} \mathrm{eV}$ corresponds to $\pi^{0}$-decay $\gamma$-ray emission, whereas the dashed and dash-dotted curves indicate the inverse Compton (IC) and Nonthermal Bremsstrahlung (NB) emissions, respectively. Right: Whipple vs RXTE flux, for the Blazar 1ES1959+650, which shows the existence of orphan $\gamma$ flares (crosses).

The connection between high energy neutrino astronomy and both gamma-ray astronomy, charged cosmic rays and gravitational waves thus emphasizes that not only a multi-wavelength but also a multi-messenger approach, combining data from different observatories, is suited for the study of the most powerful astrophysical sources in the Universe.

Sections 2 and 3 first describe the AnTARES neutrino telescope and its latest physics results. Section 4 presents the different strategies imagined to detect neutrinos from gamma-ray bursts, while section 5 details the correlations that can be performed with other observatories, such as Auger, HEss, or Virgo/LIGO. 


\section{The Antares Neutrino Telescope: principles \& description}

ANTARES is a three-dimensional grid of photomultiplier tubes, arranged in strings, able to detect the Čerenkov photons induced by the passage in sea water of relativistic charged particles, produced by the interaction of a cosmic neutrino in the Earth [5]. The measurements of the time of the hits (with a time resolution of the order of ns) and the amplitude of the hits (with a resolution of about 30\%), together with the position of the hits (by measuring the position of each PMT, to reach a resolution of about $10 \mathrm{~cm}$ ) are needed to achieve the reconstruction of those signals with the desired resolution. Muon tracks initiated by the charged current interaction of a $\nu_{\mu}$ in the Earth are detected via their directional Čerenkov light and can be reconstructed with an angular resolution below $0.3^{\circ}$ above $10 \mathrm{TeV}$. The resolution below this energy is dominated by the kinematics of the interaction. The energy resolution is quite poor, a factor 2-3 on average, restricted by the granularity/density of the light sensors and the fact that the muon traverses the detector. Showers produced by $\nu_{e}$ on the other hand emit quasi-isotropic light, and can be reconstructed with a better energy resolution (roughly $30 \%$ ) but with a poorer angular resolution, typically $3-5^{\circ}$.

The main physical backgrounds are twofold. Atmospheric muons produced in the upper atmosphere by the interaction of cosmic rays can be strongly suppressed because of their downward direction. Upward-going atmospheric neutrinos on the other hand are more delicate to identify: they have exactly the same signature as the expected cosmic signal ANTARES awaits for.

The Antares neutrino telescope, deployed at $2500 \mathrm{~m}$ below sea surface, $40 \mathrm{~km}$ off the coast of Toulon (Southern France) is composed of 12 strings, with 25 storeys each containing a triplet of 10 " photomultipliers oriented at 45 degrees downward to be optimally sensitive to upward going muons [6]. Since May 2008, 12 lines are continuously taking data. A schematic description of the detector, together with the layout of the lines, and of one of the storey, can be found in figure 2,

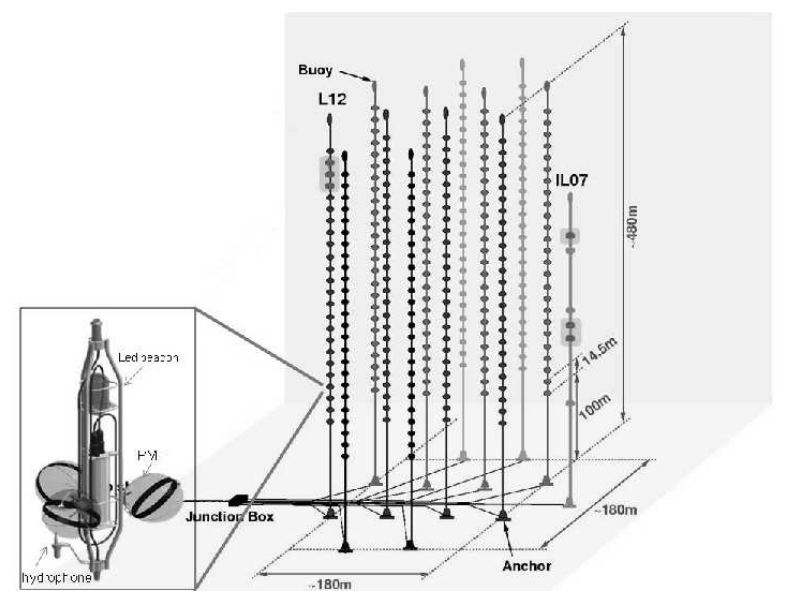

Figure 2. The layout of the completed AnTARES detector. Bottom left: optical storey with its three photomultipliers. 


\section{Selected results from ANTARES}

The main results obtained recently, related to atmospheric muons and neutrino-induced upward-going muons, are presented in this section.

\subsection{Atmospheric muons}

The attenuation of the muon flux as a function of depth can be observed in figure 3 (left), as computed using the method described in [7]. Alternatively, the reconstructed zenith angle can be converted to an equivalent slant depth through the sea water, and a depth intensity relation can be extracted, also shown in figure 3 (right). The results are in agreement with previous measurements.
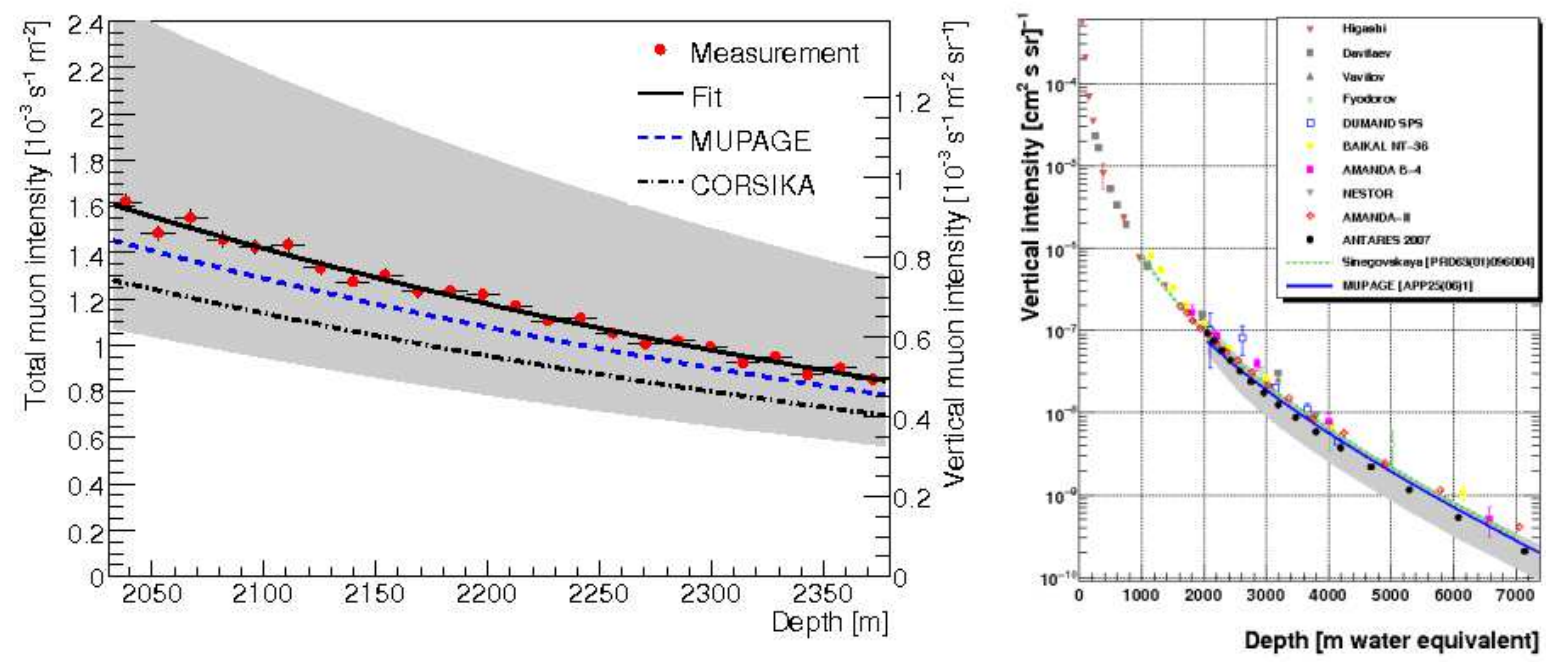

Figure 3. Left: Attenuation of the flux of muons as a function of depth, as extracted using the method described in [7. Right: Vertical depth intensity relation of atmospheric muons with $E_{\mu}>20 \mathrm{GeV}$ (black points).

\subsection{Neutrinos}

The muons produced by the interaction of neutrinos can be isolated from the atmospheric muons by requiring that the muon trajectory is reconstructed as up-going. In figure 4 the zenith angular distribution of muons in the 2007+2008 data ( 5 lines and 9-12 lines) sample is shown. A total of 1062 up-going neutrino candidates are found, in good agreement with expectations from the atmospheric neutrino background.

An all sky search, independent of assumption on the source location, has been performed on the 5-line data. No significant cluster was found. A search amongst a pre-defined list of 24 of the most promising galactic and extra-galactic neutrino sources (e.g. supernova remnants, BL Lac objects) was performed. The corresponding flux expected sensitivities, assuming an $E^{2}$ flux, are also plotted in figure 4 and compared 

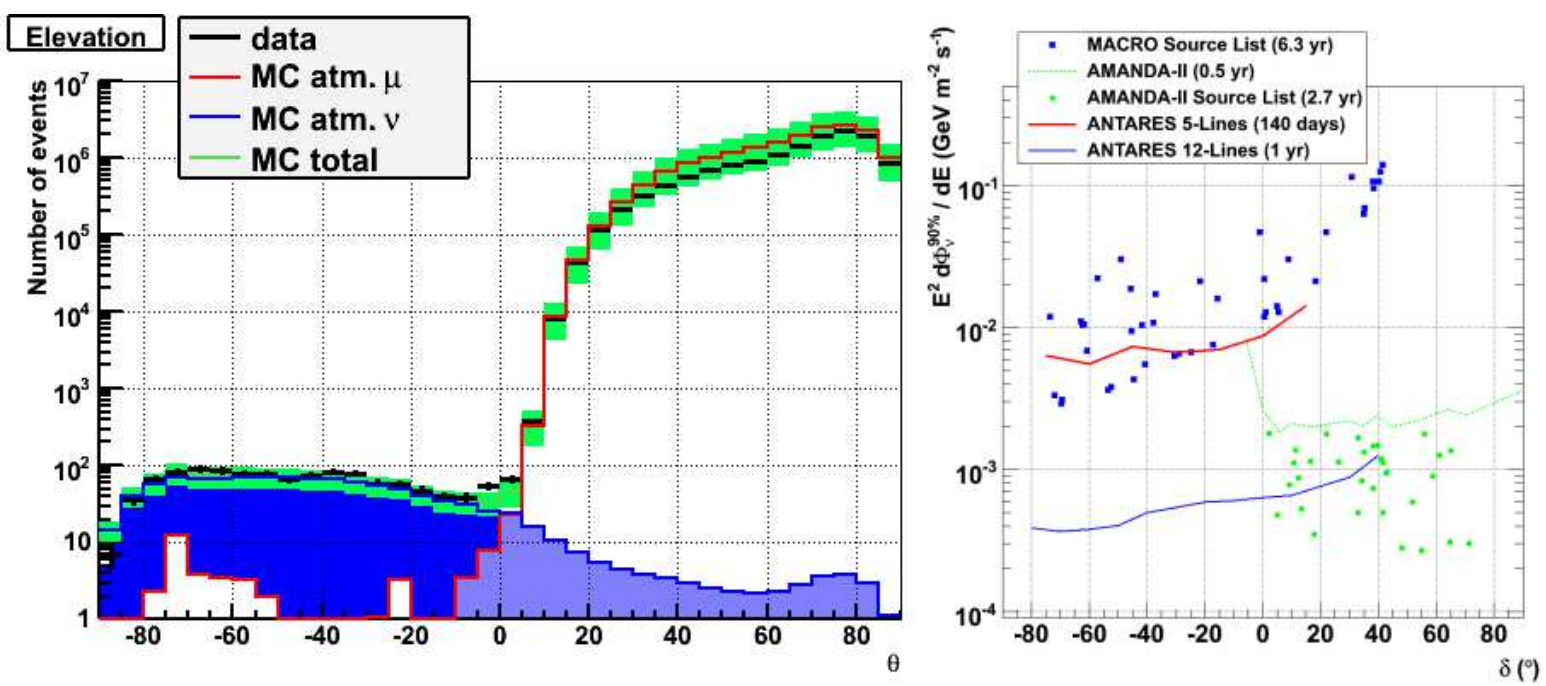

Figure 4. Left: Zenith distribution of reconstructed muons in the $2007+2008$ data. Right: expected sensitivity for ANTARES in the 5 line configuration. The expected sensitivity of ANTARES for one year with twelve lines is also shown.

to published upper limits from other experiments (see [8] for AmANDA). Also shown is the predicted upper limit for ANTARES after one full year of twelve line operation.

\section{Searches for neutrinos from GRBs}

Among all the possible astrophysical sources, transient sources offer one of the most promising perspectives for the detection of cosmic neutrinos thanks to the almost background free search. For instance, several models predict the production of highenergy neutrinos by gamma-ray bursts (GRBs) [9]: the detection of these neutrinos would provide evidence for hadron acceleration by GRBs.

Two different methods to detect transient sources can be used. The triggered search is based on the search for neutrino candidates in conjunction with an accurate timing and positional information provided by an external source. The rolling search is based on the search for high energy or multiplet of neutrino events coming from the same position within a given time window.

\subsection{Triggered search with the GCN}

Classically, GRBs or flare of AGNs are detected by gamma-ray satellites (Swift, Fermi) which deliver in real time an alert to the Gamma-ray bursts Coordinates Network (GCN, figure 5). The characteristics (direction and time of the detection) of this alert are then distributed to the other observatories. The small difference in arrival time and position expected between photons and neutrinos allows a very efficient detection by reducing the associated background. This method has been implemented in ANTARES mainly for the GRB detection since the end of 2006. Data triggered by more than 500 alerts have been stored up to now. 

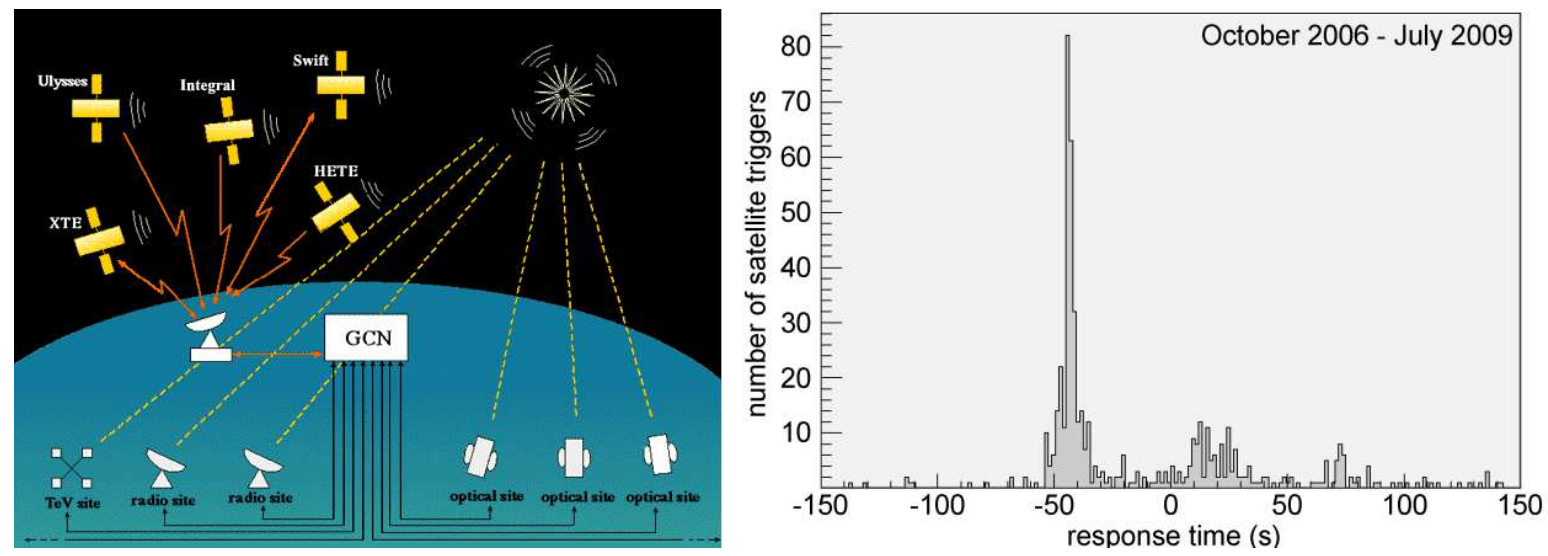

Figure 5. Left: the Gamma-ray bursts Coordinates Network (GCN). Right: ANTARES response time to an alert.

Based on the time of the external alert, in complement to the standard acquisition strategy, an on-line running program stores the data coming from the whole detector during 2 minutes without any filtering. This allows to lower the energy threshold of the event selection during the offline analysis with respect to the standard filtered data. Due to a continuous buffering of data (covering 60s) and thanks to the very fast response time of the GCN network (see figure 5), ANTARES is able to record data before the detection of the GRB by the satellite [10]. The analysis of the data relying on those external alerts is on-going.

Due to the very low background rate, even the detection of a small number of neutrinos correlated with GRBs could set a discovery. But, due to the relatively small field of view of the gamma-ray satellites (e.g., Swift has a 1.4 sr field of view), only a small fraction of the existing bursts are triggered. Moreover, choked GRBs without photons counterpart can not be detected by this method. This justifies the use of a complementary rolling search strategy.

\subsection{Rolling search: the TATOO project}

This second method relies on the detection of a burst of neutrinos in temporal and directional coincidence. Applied to Antares, the detection of 2 neutrinos within a short time is almost statistically significant: the number of doublets due to atmospheric neutrino background events is of the order of 0.004 per year when a temporal window of $900 \mathrm{~s}$ and a directional cone of $2^{\circ} \times 2^{\circ}$ are defined. It is also possible to search for single cosmic neutrino events by requiring that the reconstructed muon energy is higher than a given energy threshold (typically above a few tens of $\mathrm{TeV}$ ), for which the atmospheric neutrino background is negligible (see figure 6). When the neutrino telescope is running, this method is almost 100\% efficient and applies whenever the neutrinos are emitted with respect to the gamma flash. The main drawback is that a detection is not automatically associated to an astronomical source. It is thus fundamental to organize a complementary follow-up program to confirm the detection. 
In this context, ANTARES is organizing a follow-up program in collaboration with TAROT (Télescope à Action Rapide pour les Objets Transitoires, Rapid Action Telescope for Transient Objects), called TAToO (TAROT-AnTARES Target of Opportunity, figure 6). The TAROT network is composed of two $25 \mathrm{~cm}$ optical robotic telescopes located at Calern (South of France) and La Silla (Chile). The main advantages of the TAROT instruments are the large field of view of $1.86^{\circ} \times 1.86^{\circ}$ and their very fast positioning time (less than $10 \mathrm{~s}$ ). A GRB afterglow requires a very fast observation strategy in contrast to a core-collapse supernovae for which the optical signal will appear several days after the neutrino signal. The observational strategy is composed of a real time observation followed by few observations during the following month. Depending on the neutrino trigger settings, an alert sent to TAROT is issued at a rate of about one or two per month. The total latency is quite impressive: less than $10 \mathrm{~ms} /$ event for reconstruction, with a pointing accuracy of the order of $0.6^{\circ}$ above $10 \mathrm{TeV}$, less than 1s for alert sending, with a positioning of the telescopes in less than 10 seconds. A confirmation by an optical telescope of a neutrino alert will not
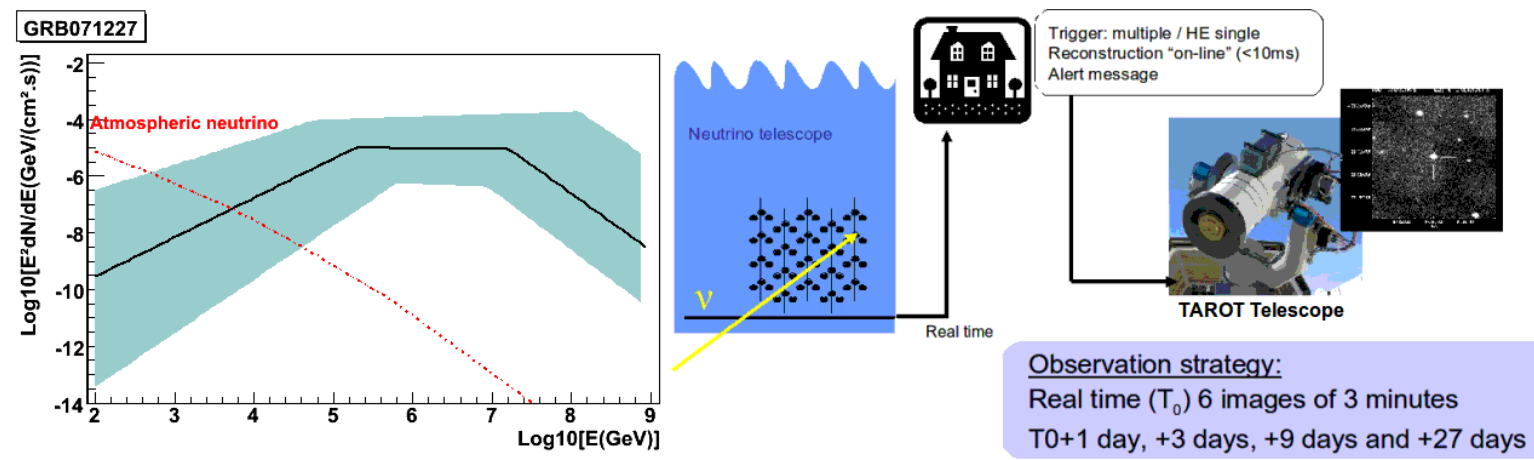

Figure 6. Left: a typical GRB neutrino spectrum. Right: Concept of TATOO.

only give the nature of the source but also allow to increase the precision of the source direction determination in order to trigger other observatories. The TAToO program is operational since February 2009, and several neutrino alerts have already been sent [11]. A torough analysis of these alerts is underway.

\section{Correlations with other observatories}

As already pointed out, high energy cosmic neutrinos could be produced in astrophysical sources that are also potential emitters of charged cosmic rays, gamma-rays or gravitational waves. Correlations with dedicated instruments could then bring a harvest of unique information about these sources.

\subsection{Auger and ultra-high energy cosmic rays}

The Pierre Auger Observatory reported an anisotropy in the arrival directions of ultrahigh energy cosmic rays (UHECR) [12. Correlation with Active Galactic Nuclei (AGN) 
from the Véron-Cetty catalog was the most significant for cosmic rays with energies higher than $57 \mathrm{EeV}$ and AGNs at distances less than $75 \mathrm{Mpc}$. The anisotropy was estimated with a confidence level of $99 \%$. The suggested correlation with local AGN sources mostly follows the location of the supergalactic plane. On the other side, no significant excess above the atmospheric neutrino flux was reported by the neutrino telescopes IceCube and ANTARES.

Instead of searching for such a localized excess, neutrino arrival direction can be correlated with the arrival directions of ultra high energy cosmic rays, as described in [13. By obtaining the probability density function of the number of neutrino events within specific angular distance from observed UHECRs, the number of neutrino events in the vicinity of observed ultra-high energy cosmic rays, necessary to claim a discovery with a chosen significance, can be calculated. For example, for 27 UHECR, a correlation significance of $5 \sigma$ is reached with $2 \%$ - $25 \%$ of neutrino events falling in $1-10^{\circ}$ bins around the original UHECR direction. Possible observed correlation of the arrival directions of those two messengers would provide a strong indication of hadronic acceleration theory.

\subsection{TeV $\gamma$ sources observed by HESS}

The exact composition of the emission of the sources observed by HEss and other gamma-ray observatories is still an open issue. Some of these sources could have a non-negligible hadronic component, and could then be neutrino emitters. In the case of continuous sources (as opposed to transients), a stacking analysis can be performed, which consists in summing up all the events detected in a given angular region around the direction of the $\gamma$ source, when below the horizon. Extended sources can be divided in different regions of interest to increase the signal-to-noise ratio. Obviously, adding more and more sources to the analysis also increases the number of background events detected. It is found that an optimum of 25 sources is needed to reach a significance close to $3 \sigma$ after 5 years of ANTARES data taking, assuming that all HESs sources are hadronic, after which the sensitivity decreases.

There have also been many discussions about the possibility to detect muons produced by high energy gamma-rays in underground, underice or underwater neutrino telescopes. In contrast to upward-going muons from neutrinos, downwardgoing muons from gamma rays suffer from a high atmospheric muon background. Therefore the sensitivity of a neutrino telescope to gamma ray induced muons is quite lower than atmospheric Cerenkov telescopes. However it monitors continuously all directions. There are at least three processes by which a photon can produce muons : photoproduction, muon pair production and charm decay. It is then possible to look for a global excess of the muon flux in a direction correlated with the position of the source (and within a given time window, if the source is transient). If the statistics is sufficient, which depends on the source spectral index and flux, accurately measured for a number of galactic sources, calibration of both the absolute pointing and the angular resolution of the neutrino telescope could be performed [14]. 


\subsection{Gravitational Waves: the GWHEN project}

Coincident searches of high-energy neutrinos (HEN) and gravitational waves (GW) are also of great interest and are detailed in [15]. Both GW and HEN are alternative cosmic messengers that carry information from the innermost regions of the astrophysical engines. Such messengers could also reveal new, hidden sources that were not observed by conventional photon-based astronomy. The observation of such coincidence would be a landmark event and can also provide observational evidence that GW and HEN originate from a common astrophysical source.

The network of GW detectors formed by the LIGO and VIRGO interferometers (figure 7) can determine the direction/time of GW bursts in connection with neutrino events observed in AnTARES. The VIRGO/LIGO network started a data-taking phase mid-2009. It monitors roughly $30 \%$ of the sky in common with ANTARES: figure 7 shows the daily averaged visibility of some sources by the network. Joint searches are ongoing within a dedicated GWHEN working group: investigations [16] led for ANTARES indicate that a joint optimized VIRGO+LIGO/ANTARES analysis is needed, in order to increase the coincident detection efficiency, while keeping the coincident false-alarm rate as low as possible, to enhance the significance of such a detection.
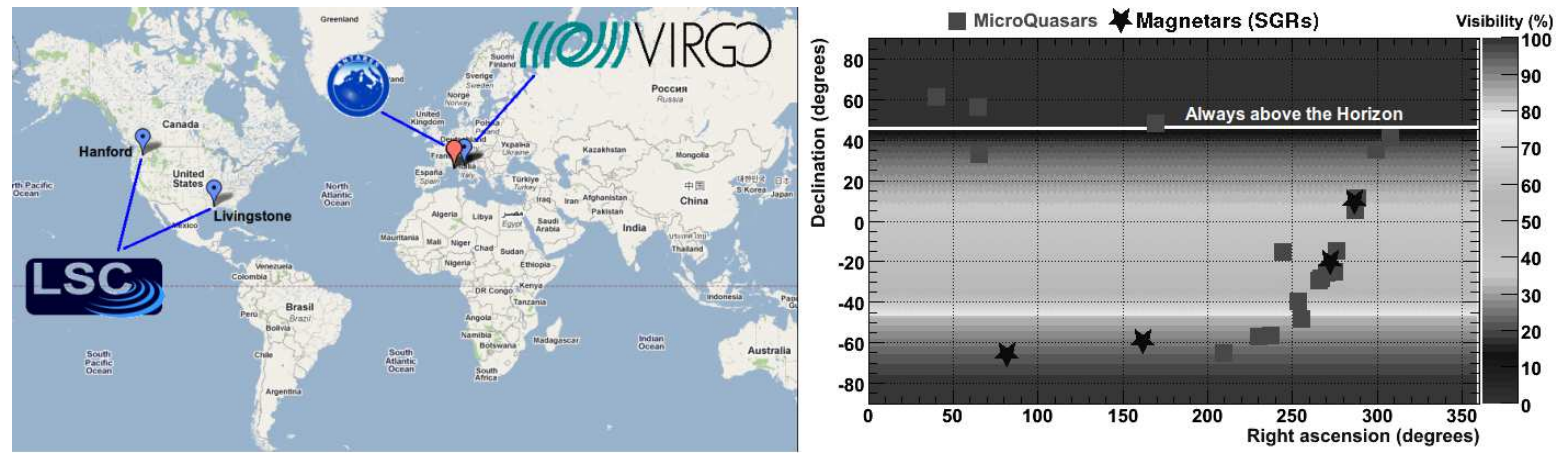

Figure 7. Left: the network of groundbased GW interferometers. The location of ANTARES is also shown. Right: averaged visibility skymap for ANTARES/VIRGO+LIGO, where the location of known microquasars/soft-gamma repeaters, potential emitters of GW bursts and HEN, is also shown. This daily (normalized) averaged visibility takes into account the interferometers' beam patterns, and the fact that a neutrino source can be detected only when below the horizon.

\section{Conclusions}

AntARES is now taking data since 2008 and has demonstrated the possibility to operate and get competitive physics results from a neutrino telescope under the sea in the Northern Hemisphere, in spite of its reduced size with respect to IceCube. It should also be noted that, because of its location, ANTAREs can observe the Galactic Centre and most of Hess sources on the galactic plane. 
Through the online and offline multi-messenger programs described hereabove, the Antares detector not only enhances its own capabilities as a neutrino telescope, but also contributes to the global effort of understanding the most violent phenomena in our Universe. In addition to offline searches for spatio-temporal correlations with other cosmic messengers (photons, cosmic rays and gravitational waves), ANTARES has the capability to handle external alerts in real time and to trigger follow-up observations with the small latency time required for the study of transient sources. The possibility to store a few minutes of raw data in coincidence with a GCN alert also brings new opportunities for offline analysis. This could be extended in the future to handle alerts involving other messengers, such as gravitational waves, to complete the GWHEN project.

Finally, the extension of the follow-up programs to other instruments in different ranges of wavelengths ( $\mathrm{X}$, radio) would undoubtely contribute to the development of the astrophysical potential of ANTARES.

Aknowledgements: Great thanks to the Organizing Committee, especially Fulvio Ricci, for trusting me for this talk.

[1] F. Halzen, for IcECuBe, Proceedings of GWDAW14 (2010)

[2] K. S. Hirata et al., Phys. Rev. Lett. 58 (1987) 1490; R. M. Bionta et al, Phys. Rev. Lett. 58 (1987) 1494 ; E. N. Alexeyev et al, PZETF 45 (1987) 461; MonT-BlanC (controversial) : V. L. Dadykin et al., PZETF 45 (1987) 464

[3] E. G. Berezhko \& H. J. Völk, arXiv:0707.4647v1, 30 th ICRC 2007, Mexico

[4] J. Holder (VERITAs Collaboration), arXiv: astro-ph/0305577v1, $28^{\text {th }}$ ICRC 2003, Japan

[5] M. A. Markov, International Conference on High-Energy Physics 578 (1960)

[6] Antares Collaboration, Nuclear Instruments and Methods in Physics Research A 555 (2005) 132

[7] Antares Collaboration, Astropart. Phys. 33 (2010) 86-90

[8] Amanda Collaboration, Phys. Rev. D 76 (2007) 042008

[9] e.g. S. Razzaque et al., Phys. Rev. D 69 (2004) 023001

[10] M. Bouwhuis, for Antares, Proceedings of $31^{\text {st }}$ ICRC conference, Lodz (2009) arXiv: astro-ph/0908.0818

[11] D. Dornic, for Antares, Proceedings of $31^{\text {st }}$ ICRC conference, Lodz (2009) arXiv: astro-ph/0908.0804

[12] Pierre Auger Collaboration, Science 318 (2007) 938

[13] J. Petrovic, arXiv:astro-ph/0908.1235

[14] G. Guillard for Antares, Proceedings of $31^{\text {st }}$ ICRC conference, Lodz (2009) arXiv: astro-ph/0908.0855

[15] E. Chassande-Mottin, for Antares/LIGO/Virgo, Proceedings of GWDAW14 (2010)

[16] Th. Pradier, Nuclear Instruments and Methods in Physics Research A 602-1 (2009) 268-274 